\title{
Focus Issue on Electrochemistry Combined with Mass Spectrometry
}

Research and applications surrounding the on-line combination of electrochemistry (EC) and mass spectrometry (MS) can be traced back as far as the early 1970s. However, this area has never been particularly main stream in either the EC or MS communities. Nevertheless, a literature search reveals that in the last decade the number of reports of EC combined on-line with MS is trending upward (Figure 1). Given the expanded research and applications of EC/MS evidenced by the increased literature in this area, it seemed an appropriate time to acquire a current snap shot of the field. In this issue of JASMS, we bring together a collection or articles, both overview and primary reports, from several different groups working on various aspects of EC/MS. These manuscripts present the reader with a picture of the potential (no pun intended!) of this combination for both fundamental and applied studies.

This focus issue contains eight papers. The first four concern the addition of a discrete electrochemical cell on-line, upstream of the ion source of the mass spectrometer. The first of these is a review article, Differential Electrochemical Mass Spectrometry by Helmut Baltruschat (Universität Bonn), that focuses on electrochemical cell design and performance for the analysis of volatile products of electrode reactions. This technique, or variations thereof, has been used for decades and finds use in important areas like catalysis research. The second paper by Hjalmar Permentier and Andries Bruins (University of Groningen), Electrochemical Oxidation and Cleavage of Proteins with On-Line Mass Spectrometric Detection: Development of an Instrumental Alternative to Enzymatic Protein Digestion, presents results that suggest on-line electrochemical techniques may be alternatives to enzymatic or chemical protein digestion methods. The third paper by Paul Gamache and co-workers (ESA, Inc.), Metabolomic Applications of Electrochemistry/Mass Spectrometry, describes some aspects of how EC may be used to complement and enhance the analytical capabilities of MS for applications relevant to clinical diagnostics, drug discovery, and other areas of biological research. The fourth paper, Ferrocenoyl Piperazide as Derivatizing Agent for the Analysis of Isocycanates and Related Compounds Using Liquid Chromatography/ Electrochemistry/Mass Spectrometry (LC/EC/MS) from Uwe Karst's group (University of Twente), reports the use of an on-line cell to oxidize (ionize) electroactive derivatives to improve analyte response.

Published online October 26, 2004
The second set of four papers covers various aspects of the electrochemical process inherent to the electrospray (ES) ion source. The paper, Negative Ion Mode Evolution of Potential Buildup and Mapping of Potential Gradients within the Electrospray Emitter by Boguslaw Pozniak and Richard Cole (University of New Orleans), describes a measurement of the interfacial potentials along the emitter electrode of the electrospray source. The magnitude of these potentials ultimately determines what reactions can take place in the emitter and at what rate. Therefore, measuring the magnitudes and determining the influences on their values are important to understanding the electrochemistry of electrospray. The next paper, Effects of Ground Loop Currents on Signal Intensities in Electrospray Mass Spectrometry by Richard Ochran and Lars Konermann (The University of Western Ontario), discusses the effect of an upstream ground current loop on the overall magnitude of the current at the electrospray emitter and the influence, or lack thereof, on the abundances and identities of ions in the ES mass spectra. Reports on "preformed ions" and analytes ionized by protonation and electron transfer are given. The paper involving a collaboration of our group at Oak Ridge National Laboratory with one from ESA, Efficient Analyte Oxidation in an Electrospray Ion Source Using a Porous Flow-Through Electrode Emitter, introduces a new type of ES emitter electrode geometry that provides high analyte electrolysis efficiency at flow

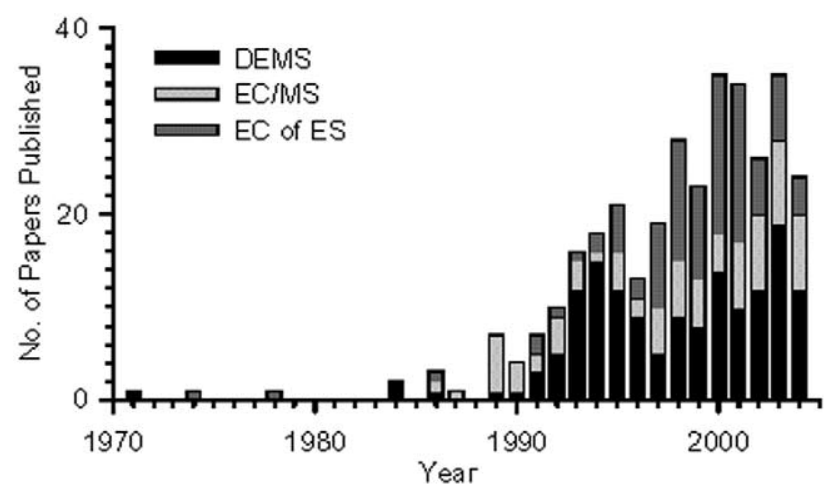

Figure 1. Rough literature search estimate of the number of papers published since 1970 on EC combined on-line with mass spectrometry. The papers were subdivided into three areas: Analysis of the volatile products of electrode reactions (differential electrochemistry mass spectrometry, DEMS), analysis of nonvolatile products using EC/MS (EC/MS, including APCI/APPI, ES, $\mathrm{PB}, \mathrm{TS}$, and ICP as ion sources), and papers dealing with the EC of the ES ion source. 
rates of several hundred microliters per minute. This capability greatly enhances the utility of the inherent EC of ES in analytical applications like analyte ionization or in fundamental investigations such as the study of the products of electrode reactions. The final paper, Generation of Mass Tags by the Inherent Electrochemistry of Electrospray for Protein Mass Spectrometry, is a review article from Hubert Girault's group (Ecole Polytechnique Fédérale de Lausanne) covering their work using the inherent EC of ES to generate electrochemically reagents that undergo homogenous reactions with analytes of interest, providing mass shifts that identify the number of free cysteines in peptides and proteins.

I thank the authors for their contributions to this focus issue and also commend the reviewers for their constructive and timely reviews. It is my hope that this issue will stimulate new ideas and expand further the growing area of EC/MS, for the benefit of mass spectrometrists, electrochemists, and the broader scientific community.

Gary J. Van Berkel Guest Editor 